\title{
The Pythagorean theorem and area formula for triangles on the plane with generalized absolute value metric
}

\section{GÖKHAN SOYDAN, YUSUF DOĞRU and N. UMUT ARSLANDOĞAN}

\section{ABSTRACT.}

In this paper, we first give the Pythagorean theorem on the plane with generalized absolute value metric and show that the converse of the Pythagorean theorem is not true in this plane. Secondly, we give necessary and sufficient conditions for a triangle in this plane to have a right angle. Finally, we give a formula for the area of a triangle on this plane.

ISIKLAR AiR FORCE High SCHOOL

16039, BURSA, TURKEY

E-mail address: gsoydan@uludag.edu.tr

E-mail address: yusufdogruleyahoo.com

E-mail address: nuarslandogan@gmail.com 